\title{
Correction to: Carbonaceous nanomaterials for phototherapy: a review
}

\section{Betül GÜRBÜZ ${ }^{1}$ • Sümeyra AYAN ${ }^{1}$ • Michael BOZLAR ${ }^{2}$ - Cem Bülent ÜSTÜNDAĞ ${ }^{1}$}

Published online: 9 September 2020

(C) Qatar University and Springer Nature Switzerland AG 2020

\section{Correction to: Emergent Materials.}

https://doi.org/10.1007/s42247-020-00118-w

The given name of the co-author Michael Bozlar was not correct, you can find the corrected name above.

The original article has been corrected.

The original article can be found online at https: //doi.org/ https://doi.org/10.1007/s42247-020-00118-w

The online version of the original article can be found at https:/doi.org/ 10.1007/s42247-020-00118-w

Cem Bülent ÜSTÜNDAĞ

cbustun@yildiz.edu.tr; cbustundag@gmail.com

1 Department of Bioengineering, Faculty of Chemical and Metallurgical Engineering, Yildiz Technical University, Esenler, 34210 Istanbul, Turkey

2 School of Engineering and Applied Science, Department of Chemical and Biological Engineering, Andlinger Center of Energy and the Environment, Princeton University, Princeton, NJ 08544, USA 